\title{
201. Rekonstruktive Maßnahmen an der Nase bei Tumordefekten
}

\author{
E. R. Kastenbauer
}

Hals-Nasen-Ohren-Klinik und Poliklinik, Freie Universität Berlin, Klinikum Charlottenburg 1 - 19, Spandauer Damm 130, D-1000 Berlin 15

\section{Reconstructive Surgery in the Nasal Region After Tumor Resection}

Summary. Successful reconstructive surgery in the nasal region requires knowledge of the technique of pedicle flap grafting, since these transplants constitute highest-grade covering material as far as their quality, thickness and colour are concerned. They are superior to free graft transplants. Various flaps taken from forehead and cheek are demonstrated. One of the most robust and universally applicable flaps for reconstruction defects of the nose and middle parts of the face is the forehead flap method of Converse because of its rich supply of blood vessels. The paper contains a detailed discussion of the various problems of this surgery.

Key words: Tumor surgery of the nose - Reconstructive surgery - Pedicle transplants.

Zusammenfassung. Eine erfolgreiche rekonstruktive Chirurgie im Nasenbereich erfordert die Kenntnis der Technik der Nahlappenplastik, da diese Transplantate aufgrund ihrer Beschaffenheit, Dicke und Farbe das hochwertigste Deckmaterial darstellen. Sie sind freien Transplantaten überlegen. Verschiedene Lappen aus der Stirn und der Wange werden demonstriert. Einer der robustesten und am vielfältigsten anwendbaren Lappen für die Rekonstruktion von Nasendefekten und des Mittelgesichtes ist der Stirnlappen nach Converse. In dem Vortrag werden die verschiedenen Probleme des subtotalen oder totalen Nasenersatzes mit gestielten Transplantaten diskutiert.

Schlüsselwörter: Tumorchirurgie der Nase - Gestielte Transplantate.

\section{Die einzeitige Wiederherstellung der Nase mit paramedianen Stirnlappen bei Defekten}

\section{G. Pfeifer}

Nordwestdeutsche Kieferklinik, Universität Hamburg, Martinistraße 52, D-2000 Hamburg 20

\section{Single-step Repair of Nasal Defects with Paramedian Forehead Flaps}

Summary. Since $1970 \mathrm{I}$ have used paramedian forehead flaps with a narrow pedicle (supratrochlear artery and vein) for nasal reconstruction in large defects in 75 patients, after the vessels had been marked by Doppler sonography preoperatively. The inner nasal lining was repaired by flaps from the margin of the defect or by fullthickness skin grafts. If the nasal skeleton is destroyed, the new soft-tissue cover is supported by angular Palacos struts prepared at our hospital and/or by autogenous or homogenous cartilage. After primary wound healing, all 75 patients had esthetically and functionally good late results. The scars on the forehead are hardly visible; there is hairless skin between the eyebrows.

Key words: Nasal reconstruction (repair) - Paramedian forehead flap - Doppler sonography - Replacement for nasal support.

Zusammenfassung. Für die Rekonstruktion der Nase bei großen Defekten habe ich seit 1970 bei 75 Patienten paramediane Stirnlappen mit dünnem Gefäßstiel verwendet (A. und V. supratrochlearis), nachdem präoperativ der Verlauf der Gefäße durch Doppler-Sonographie markiert war. Die innere Nasenschicht wurde mit Defektrandlappen oder freien Hauttransplantaten gebildet. Bei Defekten des Nasengerüstes ist die neue Weichteildecke sofort mit selbstgefertigten Winkelspänen aus Palacos und/oder autologem bzw. homologem Knorpel abgestützt worden. Bei allen 75 Patienten gab es nach primärer Wundheilung ästhetisch und funktionell die erhofften Spätergebnisse. Die Stirnnarben sind unauffällig; die Augenbrauen bleiben durch haarlose Haut getrennt.

Schliisselwörter: Nasenrekonstruktion - Paramedianer Stirnlappen - Doppler-Sonographie - Nasengerüstersatz. 